\title{
Nouvelles technologies : un levier d'amélioration de gouvernance d'entreprises en contexte Camerounais
}

\section{New technologies: a lever for improving corporate governance in the Cameroonian context}

\author{
Pierre Juselin NGUEFACK, (Doctorant) \\ Dschang School of Economics and Management, \\ Université de Dschang, Cameroun \\ Pulchérie Léonie TATANG ATABONFACK (Docteur/Ph.D) \\ Dschang School of Economics and Management, \\ Université de Dschang, Cameroun \\ Thierry joel DJATANG YAWOUO (Docteur/Ph.D) \\ Dschang School of Economics and Management, \\ Université de Dschang, Cameroun \\ Emma Joachim NYASSEU (Docteur/Ph.D) \\ Dschang School of Economics and Management, \\ Université de Dschang, Cameroun
}

\begin{abstract}
Résumé : Les nouvelles technologies engendrent un potentiel énorme pour améliorer la vie et la survie des entreprises en général et des personnes dans les pays en développement en particulier. L'objectif de cette étude est de mesurer l'effet des nouvelles technologies sur la gouvernance des entreprises au Cameroun. Pour atteindre notre objectif, nous avons eu recours d'une part, à une démarche hypothético-déductive, l'échantillonnage par choix raisonné et d'autre part, à une régression logistique pour tester les hypothèses de la recherche. Les résultats obtenus auprès d'un échantillon de 90 entreprises ayant le statut de SA et SARL relèvent d'abord que les nouvelles technologies (utilisation des visioconférences, et la mise en réseaux des ordinateurs) ont des effets positifs et statistiquement significatifs non seulement sur la fréquence des réunions du conseil d'administration mais aussi sur la présence des investisseurs institutionnels. Cependant, leur influence est plutôt négative et statistiquement significative sur la durée de mission de l'audit externe.
\end{abstract}

Mots clés : innovations numériques, gouvernance d'entreprise, SA, SARL.

\begin{abstract}
New technologies have enormous potential to improve the lives and survival of businesses in general and people in developing countries in particular. The objective of this study is to measure the effect of new technologies on corporate governance in Cameroon. To achieve our objective, we used a hypothetical-deductive approach, purposive sampling, and logistic regression to test the research hypotheses. The results obtained from a sample of 90 companies with the status of SA and SARL show, first of all, that new technologies (use of video-conferencing and computer networking) have positive and statistically significant effects not only on the frequency of board meetings but also on the presence of institutional investors. However, their influence is rather negative and statistically significant on the duration of the external audit.
\end{abstract}

Keywords: digital innovations, corporate governance, SA, SARL.

Digital Object Identifier (DOI): https://doi.org/10.52502/ijesm.v1i3.204 


\section{Introduction}

Les nouvelles technologies engendrent un potentiel énorme pour améliorer la vie des entreprises en général et des personnes dans les pays en développement en particulier. L'utilisation des technologies numériques peut stimuler le commerce, appuyer les systèmes d'éducation et de santé et renforcer à tous les niveaux les gouvernements dans leur processus de développement à l'échelle globale. De nos jours, il est difficile d'imaginer nos vies sans l'ordinateur parce qu'il est partout présent autour de nous, dans les voitures, les téléphones, les avions, les banques, les écoles, etc.

En effet, les nouvelles technologies, comme facteur de changement technologique, représentent une révolution numérique qui est d'ores et déjà une réalité dans les pays développés et les BRIC (Brésil, Russie, Inde, Chine) et commence à se diffuser dans les autres pays émergents ouvrant des perspectives de croissance entraînant de profondes mutations organisationnelles et managériales (Bloom et Van Reenen, 2010 ; Van Reenen et al., 2010 ; OCDE, 2009, 2005 ; Black et Lynch, 2004, 2001 ; Brynjolfsson et Hitt, 2003 ; Bresnahan et al., 2002 ; Caroli et Van Reenen, 2001). Dans cette optique, les innovations de rupture générées par les nouvelles technologies permettent aux entreprises de renforcer leurs avantages concurrentiels en se différenciant de leurs concurrents ; plus précisément, elles créent un capital organisationnel basé sur des partenariats industriels et financiers (Braune, Lantz, Sahut et Teulon, 2019). Elles contribuent également au developpement économique dans son ensemble (El Bouame et Lempqeddem, 2021). De ce fait, la transformation numérique des entreprises est un parcours qui consiste à adopter et à déployer des technologies et des modèles commerciaux numériques afin d'améliorer les performances de manière quantifiable. Ainsi, les transformations numériques modifient les modèles commerciaux, la création de valeur et peuvent influencer positivement la réputation d'une entreprise (Anderson, 2014). Cependant, les attentes générées par ces opportunités technologiques sont susceptibles de générer des dépenses toujours plus importantes qui dépassent leurs bénéfices réels. Comme le soulignait Solow (1987) lors de la révolution informatique, «il faut faire attention » et la question de la mesure des gains liés aux transformations numériques se pose donc.

Les nouvelles technologies telles que l'intelligence artificielle, les médias sociaux, les logiciels et le matériel à source ouverte, le crowdsourcing, la confiance électronique et l'évaluation de la réputation en ligne, la signature électronique, la réalité virtuelle, la réalité augmentée, le zoom, l'IdO, l'impression 3D, l'imagerie numérique et les données volumineuses donnent du pouvoir aux futurs entrepreneurs, tout en modifiant radicalement le paysage concurrentiel et en contribuant à réduire considérablement les barrières entre l'invention et la création d'une nouvelle entreprise (Kelly, 2016). Ces technologies facilitent le traitement rapide et décentralisé de l'information (Czernich et al., 2011, Bloom et Van Reenen, 2007). Leur utilisation permet la diffusion des informations, renforce la productivité des employés (Aral et al., 2007) et améliore les niveaux de performance des entreprises 
(Bloom et al., 2010, 2006, 2005 ; Badescu et Garcés-Ayerbe , 2009 ; Eurostat, 2008 ; OCDE, 2008, 2004 ; Motohashi, 2008, 2007 ; Draca et al., 2006 ; Atrostic et Nguyen, 2005, 2002 ; Arvanitis, 2004 ; Hollenstein, 2004 ; etc.). Elles contribuent également à atténuer les problèmes de gouvernance d'entreprise et les facteurs de risque connexes, qui entravent gravement le développement de la microfinance, en commençant par une application utile de la double vérification des emprunts multiples non déclarés et autrement difficilement détectables. Il y a donc une lutte prospective pour rendre compte et comprendre pleinement ces nouvelles tendances (Sahut, Dana et Laroche, 2019).

Notons que l'utilisation des nouvelles technologies dans les entreprises a fortement augmenté au fil du temps. La technologie a déjà un impact perturbateur sur les mécanismes de gouvernance des entreprises, en particulier dans les pays moins sophistiqués (Claessens et Yurtoglu, 2013). L'adoption de ces nouvelles technologies dans notre quotidien nécessitera encore un temps d'adaptation et de transition. Mais le confinement imposé par la propagation de la Covid-19 aura été un réel accélérateur à l'adoption du télétravail pour de nombreuses entreprises qui ne l'utilisaient pas jusqu'à présent. Si les outils n'étaient pas en place dans beaucoup de structures, le confinement aura permis de franchir le pas et de l'instaurer définitivement sur le long terme.

Les chercheurs ont utilisé la théorie de l'organisation industrielle afin d'examiner comment les entreprises interagissent entre elles dans les décisions d'investissement dans les nouvelles technologies et comment les avantages qui en résultent sont divisés. La théorie des jeux a été utilisée pour examiner le rôle de l'interaction stratégique entre les entreprises concurrentes dans l'adoption des nouvelles technologies (Belleflamme, 2001). D'autres chercheurs ont utilisé la théorie de l'agence (Bakos et Nault, 1997; Clemons et Kleindorfer 1992). La théorie des coûts de transaction a également complété la compréhension du rôle des technologies numériques dans la réduction des coûts de transaction (Clemons et Row, 1991; Gurbaxani et Whang, 1991). Dans la théorie du changement technologique, le processus de décision a une incidence sur l'adoption de nouvelles technologies (Pennings, 1987; Lefebvre 1992). Pour Adeosun et al., (2009), l'utilisation des nouvelles technologies dans les entreprises permet la gestion stratégique, la communication, la collaboration, l'accès à l'information, la prise de décision, la gestion des données et la gestion des connaissances. Dans une étude menée auprès de 200 une PME britanniques, Maguire et Koh (2007) ont observé que les PME pourraient acquérir un avantage concurrentiel avec l'utilisation des technologies numériques. Leur enquête a révélé que 70 pour cent des PME ont acquis un avantage concurrentiel sur le marché mondial grâce aux nouvelles technologies. Ces technologies permettent de réduire les coûts et pourraient être utilisé pour améliorer les processus internes et une communication plus rapide avec les clients (Kotelinkov, 2007).

Ainsi, les enjeux soulevés par l'usage des nouvelles technologies pour l'amélioration de la gouvernance d'entreprise ont généré une littérature peu abondante. Si certaines études ont souligné l'effet négatif des nouvelles technologies sur l'entreprise (Freeman et Soete, 1997 ; Aghion et Howitt, 
1998), d'autres montrent leur effet positif sur la gouvernance et la croissance, ainsi que sur la capacité des pays à bénéficier de la mondialisation (Timmer et van Ark, 2005 ; Kretschmer, 2012). Cet article vient combler cette lacune. L'objectif principal de cette étude est donc de mesurer l'effet des nouvelles technologies sur la gouvernance d'entreprise au Cameroun. De surcroit, nous vivons dans une ère où les technologies prennent de plus en plus de place dans notre quotidien. Big data, intelligence artificielle, IoT, blockchain... des mots qui paraissaient encore barbares il y a quelques années, ont toute leur place aujourd'hui au sein des outils numériques. C'est pourquoi à l'heure où une pandémie touche le monde entier, il est intéressant de se demander quelle est l'effet des nouvelles technologies sur la gouvernance d'entreprise?

La suite de cet article est organisée autour de quatre points principaux. Le deuxième point présente La gouvernance d'entreprise à l'épreuve des nouvelles technologies. Le troisième point expose la démarche méthodologique. Le quatrième point présent les Résultats et Discussions. Le cinquième point énonce la conclusion et Recommandations.

\section{La gouvernance d'entreprise à l'épreuve des nouvelles technologies}

Il est question d'examiner d'une part la diffusion et l'adoption des nouvelles technologies sur les mécanismes de gouvernance (I.1), et d'autre part la fracture numérique inter entreprise sur changement organisationnel (I.2).

\subsection{De la diffusion à l'adoption des nouvelles technologies par les organisations}

Dans leurs travaux, Fors et Stolterman (2004) ont décrit l'innovation numérique comme «des changements que la technologie numérique entraîne ou influence dans tous les aspects de la vie humaine». De leur côté, Leger et al. (2017) affirment que la nouvelle technologie est l'émergence d'un nouveau type d'innovation. Ces innovations numériques apportent un grand changement au sein des organisations dans tous les domaines. Les innovations numériques qui concernent le développement et la mise en œuvre d'artefact innovante et de solutions connexe qui sont basées sur la réutilisation innovante des composantes numériques (Fichman et al., 2014 ; Yoo et al. 2010). Des recherches extérieures ont montrés que l'élément caractéristique des innovations numériques est l'utilisation de technologies numériques innovantes de manière inédite (Kohli et Melville, 2018 ; Nambisan et al., 2017).

Le point de départ théorique de notre analyse est la littérature sur l'adoption des nouvelles technologies. Cette littérature explique entre autre les facteurs d'adoption de nouvelles technologies et des différences dans les taux d'adoption entre les entreprises, les industries et les pays (Geroski, 2000). L'adoption des technologies numériques constitue une opportunité d'amélioration de la compétitivité des entreprises des pays en développement (Aubert et al., 2009 ; Gnansounou, 2010 ; Missoui, 2011 ; Steinmueller, 2001 ; Lal, 2004 ; Basant et al., 2011 ; Tello, 2011 ; Calza et Rovira, 2011 ; Gutiérrez, 
2011 ; Akomea-Bonsu, 2012). Ainsi pour comprendre l'adoption et la diffusion des nouvelles technologies dans les entreprises des PED, il est donc essentiel de découvrir les facteurs qui expliquent le retard et la variation du taux d'adoption. Les modèles théoriques existants se concentrent sur un certain nombre de facteurs expliquant ce retard et la variation du taux d'adoption, notamment: l'incertitude sur les caractéristiques de la nouvelle technologie (Jensen, 1982), des considérations stratégiques, comme les différences dans les taux de profit avant et après l'adoption de la technologie numérique en fonction de la structure du marché (Reinganum, 1981), les processus d'apprentissage par la pratique (Jovanovic et MacDonald, 1994) et les différences dans le capital humain (Nelson et Phelps, 1966; Chari et Hopenhayn, 1991 ). Helpman et Trajtenberg (1998) ont analysé l'adoption des nouvelles technologies et ont montré que l'adoption de ces technologies est plus rapide quand la croissance de la productivité est élevée par rapport à celle de l'ancienne technologie.

La littérature soutient l'idée que la nouvelle technologie (NT) augmente la capacité de collecter, de traiter et de diffusion des informations grâce au comportement des acteurs, à leur interaction et collaboration. Elles permettent une meilleure diffusion d'information et un partage plus aisé entre les acteurs de l'organisation (R. Reix.1998). L'introduction de l'innovation numérique au sein des entreprises s'observe sur le changement organisationnel et l'utilisation des nouveaux procédés. Disle et Noël (2007), avancent que l'innovation numérique permet d'améliorer le système d'information comptable. Al-Majali (2011) applique ce modèle en y intégrant le big data, l'intelligence artificielle, et la technologie blockchain pour étudier leurs effets sur la gouvernance d'entreprise. Il révèle que ces trois variables intégrées sont des facteurs significatifs ayant des influences sur le contrôle de l'entreprise. Ainsi, il conclut que la technologie numérique influence positivement le fonctionnement du conseil d'administration et de l'audit externe. Dans le même ordre d'idée, les résultats de l'étude de (Zolait et Ainin, 2010) montrent que le digital facilite le fonctionnement et le contrôle des entreprises.

Par ailleurs, Luarn et Lin, (2005) ont utilisé la theorie de l'acceptation pour expliquer l'effet de la nouvelle technologie sur les mécanismes de gouvernance d'entreprise. Pour ses auteurs, l'utilisation de l'intelligence artificielle, de technologie blockChain et du big data influencent positivement le fonctionnement du conseil d'administration, et la structure de propriété. Par ailleurs, Amin et al. (2008) suite à une étude menée auprès des entreprises en Malaisie, confirment l'influence des variables de la nouvelle technologie (intelligence artificielle, la technologie blockChain, le big data, etc...) sur la fréquence de réunions du conseil d'administration, et l'appartenance de l'audit externe.

\subsection{De la fracture numérique inter entreprise au Changement organisationnel}

Dans ses travaux, Benchenna (2012) démontre une corrélation direct entre les nouvelles technologies et la croissance économie qu'il qualifie de fracture numérique ». Dans cette logique, 
Fulssack et al. (2005) soulignent que la fracture numérique est le fossé entre ceux qui utilisent les potentialités des nouvelles technologies pour leur accomplissement personnel ou professionnelle et ceux qui ne sont pas en état de les exploitent, faute d'accès aux équipements et de sensibilisation ou de formation pour les maitrisées convenablement. La fracture numérique revête différentes formes et concerner à la fois l'accès, l'usage, le contenu et la prise de décision.

De ce fait, nous notons que la fracture numérique est une barrière ou encore une fosse numérique qui existe entre les individus, des groupes sociaux ou des états. Cette fosse peut être à l'échelle internationale, c'est-à-dire entre les pays développés et ceux en développement ou à l'échelle nationale c'est-à-dire a l'intérieur même d'un pays (l'intérieur même des sociétés, entre les milieux urbains et périphérique y compris, aux seins des classes sociales). Ainsi, la fracture numérique se perçoit de plus en plus sous l'angle de clivage socio-démographie (entre les individus, les ménages, les entreprises, et aires géographique), de disparité socio-économie (l'accès aux technologies numériques et le coût de la connexion à l'internet) et de appropriation technique [vodoz, 2010 ; Soupizet, 2004].

Les nouvelles technologies influencent sur des divers aspects de l'entreprise même si les études empiriques manquent encore pour estimer l'application effective de la technologie disruptive sur la gouvernance d'entreprise (Esposito De Falco et Cucari, 2018). Cependant, Yermack (2017) a tenté d'étudier l'effet de la technologie disruptive sur la gouvernance d'entreprise. La technologie de la chaîne de blocage peut simplifier l'adhésion des entreprises aux récents changements réglementaires introduits par la directive sur le droit des actionnaires (directive européenne 2017/828) grâce à une identification plus directe des personnes ayant le droit de vote, à une plus grande transparence des procédures et des mécanismes de vote des investisseurs institutionnels et de leurs fournisseurs de services.

Les nouvelles technologies activement sont mises en œuvre pour modifier l'essence même de la gouvernance d'entreprise afin d'accélérer les processus décisionnels, d'améliorer la transparence, la prévision des risques et les résultats des décisions. Par ailleurs, une étude à grande échelle menée par le Conseil international d'experts du Forum économique mondial (2015), affirme que 75,4 \% des PDG interrogés pensent que $30 \%$ des audits d'entreprise seront réalisés par intelligence artificielle d'ici 2025. En outre, 45,2\% des répondants pensent que, le conseil d'administration pourrait inclure dans le processus de contrôle et de gestion un premier robot doté d'intelligence artificielle pour la prise de décision d'ici (2025 Schwab K. 2016).

La littérature laisse paraitre que grâce aux nouvelles technologies, il sera possible de modifier les erreurs humaines et les conflits d'intérêts qui pourraient entrainer une mauvaise la gouvernance d'entreprise. La mise en œuvre de l'intelligence artificielle est particulièrement importante pour le contrôle de l'environnement interne et externe de l'entreprise (Nikishova, 2017). A cet effet, Blackrock (2018) soutient que la présence des caméras de surveillance et des forums influence 
positivement le fonctionnement du conseil d'administration. Dans un même ordre d'idée, Heidrick et Struggles, (2014) démontrent que «les conseils d'administration du monde entier se concentrent de plus en plus sur la conformité plutôt que sur l'excellence ». L'ère numérique a déjà un impact considérable sur la société et les organisations (Ko et Fink, 2010 ; Newell et Marabelli, 2015). Selon Kaal (2017), la technologie blockchain pourrait conduire à un modèle de gouvernance décentralisée dans lequel les mécanismes classiques de contrôle interne et externe nécessaires pour résoudre les problèmes de gouvernance d'entreprise. Dans le même ordre d'idées, Lafarre et Van der Elst, (2018) soutiennent que la technologie blockchain pourrait offrir une solution pour l'amélioration de la gouvernance d'entreprise.

De l'ensemble de ce developpement, nous émettons les hypothèses suivantes:

\section{H1 : L'utilisation des nouvelles technologies augment la fréquence des conseils d'administration.}

\section{H2 :L'utilisation des nouvelles technologies favorise l'attraction des investisseurs institutionnels.}

La littérature sur la gouvernance perçoit généralement l'audit comme un mécanisme de gouvernance permettant d'éviter les conflits potentiels entre actionnaires et gestionnaires de garantir la divulgation d'informations fiables sur les comptes (Carcello et al. 2011). Pour Najoua et Baudier (2017) l'utilisation du logiciel informatique au sein de l'entreprise influence positivement sur la mission de l'auditeur externe.

Par ailleurs, Wallace (2004) ; Andon et al. (2014); Jeacle (2014, 2017 ) affirment que l'utilisation du logiciel (ERP) dans l'entreprise facilite la vérification des comptes de l'entreprise. Cette présence du logiciel informatique n'influence pas sur les honoraires de l'auditeur externe. Pour eux, l'entreprise ressource planning (ERP) permet aux auditeurs externes de réduire le risque de publication de fausses informations aux actionnaires. Manita et Patricia (2017) soutiennent que la présence d'un site internet permet l'auditeur externe d'avoir d'autres informations sur les comptes de l'entreprise. Selon Georges Ataya (2018) le big data améliore la protection des informations de l'entreprise et les sevices de l'auditeur externe.

\section{H3 : Les nouvelles technologies réduisent la durée de mission de l'audit externe.}

\section{Méthodologie}

\subsection{La collecte des Données}

La collecte des données s'est faite par une enquête de terrain, faute d'une base de données portant sur les technologies numériques dans les entreprises au Cameroun. Ainsi, la collecte de ces données s'est faite par entretien direct à partir d'un questionnaire administré auprès de 90 l'ensemble des entreprises ayant le statut juridique de SA ou SARL au Cameroun entre Mai et Juin 2021. Ce nombre se justifie par le fait qu'une même entreprise dans deux villes différentes ne devrait pas figuré simultanément dans l'échantillon, puisqu'en général, les stratégies adoptées en matière de technologie 
numérique ne changent pas d'une agence à l'autre au sein du même groupe. Par ailleurs, la phase d'enquête a permis de constater que plus du tiers des entreprises n'étaient pas localisées à l'adresse officielle, d'où cette taille de échantillon.

\subsection{Sélection et mesure des variables}

La gouvernance d'entreprise est appréhendée à travers trois dimensions : le conseil d'administration, la structure de propriété, et l'audit externe.

Cependant, la nouvelle technologie est captée par :

L'intelligence artificielle qui est selon Winston(1992), « l'étude des mécanismes permettant à un agent de percevoir, raisonner et agir ». (Elle repose sur variables à savoir : La robotique est l'ensemble des techniques permettant la conception et la réalisation des machines automatiques ou de robots. La vidéoconférence permet à deux ou plusieurs interlocuteurs physiquement sépares de se voir s'écouter et parler en temps réel, cette possibilité offre évidemment de nombreuses applications dans l'enseignement à distance ; les conférences et débats avec des experts, les étrangers entre correspondants étrangers, les projets collaboratif et diverses autre activités).

> La technologie blockChain qui est une technologie de rupture ayant le potentiel de transformer les fondements de nos systèmes sociétaux et économiques, en utilisant des termes comme, nouvel internet, internet de confiance ou internet de valeur.

$>$ Le Big Data qui est très régulièrement défini sous le concept des 5V voir (Vasarhelyi et al., 2015; Warren et al., 2015) : Volume, Vitesse, Variété, Véracité et Valeur. La définition donnée au Big Data est relative et varie d'une entreprise à une autre.

Tableau 1: Opérationnalisation de la variable dépendante (la gouvernance d'entreprise)

\begin{tabular}{|l|l|l|l|}
\hline \multicolumn{2}{|c|}{ Variables } & \multicolumn{1}{|c|}{ Auteurs } & Mesure des variables \\
\hline $\begin{array}{l}\text { Conseil } \\
\text { d'administration }\end{array}$ & $\begin{array}{l}\text { Fréquence des réunions } \\
\text { (FREQ_REU) }\end{array}$ & $\begin{array}{l}\text { Godard et Schatt (2004); } \\
\text { Andres et al. (2005) }\end{array}$ & $\begin{array}{l}\text { Mesurer par le nombre de réunions du } \\
\text { conseil par an }\end{array}$ \\
\hline $\begin{array}{l}\text { Structure de } \\
\text { propriété }\end{array}$ & $\begin{array}{l}\text { Propriété des investisseurs } \\
\text { institutionnels(PRO_INST }\end{array}$ & $\begin{array}{l}\text { Healy et al. (1999); Ajinkya et } \\
\text { l. (2004); Xiao et al. (2004); } \\
\text { Ducassy (2008) }\end{array}$ & $\begin{array}{l}\text { Variable binaire égale 1 s'il existe des } \\
\text { investisseurs institutionnels et 0 si non }\end{array}$ \\
\hline Auditeur externe & $\begin{array}{l}\text { La durée de la mission de } \\
\text { l'audit externe (DU_MIS) }\end{array}$ & $\begin{array}{l}\text { Shockley et Holt (1983); De } \\
\text { Angelo (1981) }\end{array}$ & $\begin{array}{l}\text { Variable nominale } \\
\text { Les honoraires d'audit (LN } \\
\text { HAUD) }\end{array}$ \\
$\begin{array}{l}\text { Carcello et al. (2002); Mitra et } \\
\text { al. (2007) }\end{array}$ & $\begin{array}{l}\text { Logarithme népérien du montant des } \\
\text { honoraires d'audits }\end{array}$ \\
\hline
\end{tabular}

Source: nous-mêmes 
Tableau 2 : Opérationnalisation de la variable indépendante

\begin{tabular}{|c|c|c|c|}
\hline Variables & & Auteurs & Mesures \\
\hline \multirow{3}{*}{ L'intelligence artificielle } & $\begin{array}{lll}\text { L'utilisation } & \text { de } & \text { la } \\
\text { robotique(ROB) } & & \end{array}$ & $\begin{array}{l}\text { Christoph (2016); } \text { Yermack } \\
(2017) \text {; Lafarret (2018) }\end{array}$ & \multirow{3}{*}{$\begin{array}{l}\text { Variable binaire } 1 \text { - } \\
\text { oui 2- non }\end{array}$} \\
\hline & $\begin{array}{l}\text { Camera } \\
\text { sécurité(CAM_SECU) }\end{array}$ & \multirow[t]{2}{*}{\begin{tabular}{llll} 
Hui $\quad(2014) ;$ & \multicolumn{2}{l}{ OCDE(2016); } \\
Lakhani $\quad(2013) ;$ & Mc & Kinsey \\
$(2017)$ & & &
\end{tabular}} & \\
\hline & $\begin{array}{lr}\text { L'utilisation } & \text { des } \\
\text { visioconférences(VISIO_CONF) }\end{array}$ & & \\
\hline La technologie blockchain & $\begin{array}{l}\text { Ordinateur } \quad \text { en réseau } \\
(\mathrm{ORD} \text { RES) et employés en } \\
\text { réseau (EMP_RES) }\end{array}$ & $\begin{array}{lcll}\text { Lafarret (2018); } & \text { Porter } & \text { et } \\
\text { Heppelmann (2018) } & & \end{array}$ & $\begin{array}{l}\text { Variable binaire 1- } \\
\text { oui 2- non }\end{array}$ \\
\hline \multirow{3}{*}{ Le big data } & $\begin{array}{l}\text { La plateforme } \\
\text { communication (PLA_COM) }\end{array}$ & \multirow{3}{*}{$\frac{\frac{\text { Wallace }(2004)}{(2014) ; \text { Jeacle }(2014,2017)} ; \frac{\text { Andon et al. }}{\text { Manita et Patricia }(2017)}}{\text { (2017) }}$} & \multirow{3}{*}{$\begin{array}{l}\text { Variable binaire } 1- \\
\text { oui 2- non }\end{array}$} \\
\hline & $\begin{array}{lll}\text { Logiciel } & \text { de } & \text { contrôle } \\
(\text { LOG_CON }) & & \end{array}$ & & \\
\hline & Site internet (SI_INT) & & \\
\hline
\end{tabular}

Source: nous-même à partir de la littérature

Tableau 3 : Variable de contrôle

\begin{tabular}{|l|l|l|}
\hline Variable de contrôle & Auteur et référence & Mesures \\
\hline Effectifs du personnel & Pearce et Zahra (1989); & Mesure par la variable ordinaire \\
\hline Secteur d'activité & $\begin{array}{l}\text { Gillan et Starks (2003); Mitra et } \\
\text { al. (2007) }\end{array}$ & $\begin{array}{l}\text { Variable binaire qui prend un si l'entreprise } \\
\text { appartient à un secteur des hautes technologies, } \\
\text { zéro dans le cas inverse. }\end{array}$ \\
\hline
\end{tabular}

Source : Nous même à partir de la littérature

Etant donné que cette étude permet de mesurer l'effet des technologies numériques sur la gouvernance des entreprises au Cameroun le modèle ci-après a été adopté :

$$
\text { GOUVER_ENT }=\boldsymbol{\beta}_{\mathbf{0}}+\boldsymbol{\Sigma}^{\mathrm{n}_{\mathrm{i}=1}} \boldsymbol{\beta}_{\mathrm{i}} \mathbf{X i}+\boldsymbol{\mu}_{\mathrm{i}} \quad \text { avec : }
$$

GOUVER_ENT désigne la gouvernance d'entreprise. Elle représente la variable dépendante ou variable à expliquer. $\beta 0$, le terme constant ; $\beta \mathrm{i}$, les coefficients des facteurs expliquant la gouvernance d'entreprise; Xi, les variables décrivant les innovations numériques dans les entreprises ; $\mu_{\mathrm{i}}$, le terme d'erreur. La forme empirique complète du modèle est :

\section{GOUVER_ENT $=\beta_{0}+\beta_{1}$ ROB $_{\mathrm{i}}+\beta_{2}$ CA_SUV $i \mathrm{i}+\beta_{3}$ VIS_CON $\mathrm{i}+\beta_{4}$ ORD_RES $_{\mathrm{i}}+\beta_{5}$ PLA_COM +

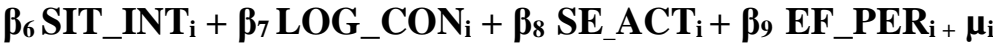

Nous allons estimer ce modèle par une régression logistique de type multinomiale pour la simple raison que la variable dépendante gouvernance d'entreprise est composée de plus de deux 
modalités $(k>2)$ qui sont des variables qualitatives. En plus, cette estimation sera faite en fonction de plusieurs autres variables explicatives de l'innovation numérique sont également de nature qualitatives.

\subsection{Outils statistiques d'analyse}

En ce qui concerne outils statistiques d'analyse, nous avons utilisé les tests de corrélation de Pearson, le teste de Khi-deux et une régression logistique de type multinomiale. Le test de corrélation étant une mesure d'association qui permet d'établir si deux variables mesurées sur le même ensemble d'observation varie de façon analogue ou non et permet également de voir s'il y a un problème de multi colinéarité entre les variables. Pour ce qui est du test de Khi-deux, le but de tester l'existence d'une relation statistique entre deux variables et le cas échéant d'établir la forme de cette relation.

En ce qui concerne la régression logistique de type multinomiale, elle permet d'expliquer une variable dépendante et de nature métrique en fonction de plusieurs autres variables dites explicatives. Cette analyse a un avantage car prend en compte les interrelations pouvant exister entre les variables explicatives.

\section{Résultats et Discussions}

Cette section qui présente les résultats de l'étude est organisée autour de deux points : la première porte sur le test de corrélation. Le deuxième présente les résultats des régressions

\subsection{Test de multi colinéarité sur les variables}

Avant de présenter les résultats de la régression, il est nécessaire de présenter la matrice de corrélation, question de contrôler s'il existe un problème sérieux de multi colinéarité entre les variables. Cette matrice se présente comme suit :

\section{Tableau 4: matrice de corrélation}

\begin{tabular}{|c|c|c|c|c|c|c|c|c|}
\hline & ROB & $\begin{array}{l}\text { CAM_SU } \\
\mathrm{R}\end{array}$ & $\begin{array}{l}\text { VISIO_CON } \\
\text { F }\end{array}$ & ORD_RES & EMP_RES & PLA_COM & $\begin{array}{l}\mathrm{LOG}_{-} \\
\mathrm{CON}\end{array}$ & $\overline{\text { SIT_INT }}$ \\
\hline ROB & 1 & & & & & & & \\
\hline CAM_SUR & 0,266 & 1 & & & & & & \\
\hline VISIO_CONF & 0,164 & 0,424 & 1 & & & & & \\
\hline ORD_RES & 0,104 & $-0,023$ & $-0,046$ & 1 & & & & \\
\hline EMP_RES & $-0,019$ & $-0,128$ & $-0,103$ & 0,362 & 1 & & & \\
\hline PLA_COM & $-0,027$ & $-0,098$ & $-0,164$ & $-0,074$ & 0,075 & 1 & & \\
\hline LOG_CON & $-0,043$ & $-0,123$ & $-0,035$ & $-0,081$ & $-0,101$ & 0,160 & 1 & \\
\hline SIT_INT & 0,057 & $-0,066$ & $-0,053$ & $-0,008$ & 0,047 & 0,245 & 0,207 & 1 \\
\hline
\end{tabular}

Source : Auteur à partir de SPSS version 20 
Le tableau ci-dessus met en relief les coefficients de corrélation de Pearson calculés pour l'ensemble des variables de l'étude. L'analyse de la matrice des coefficients montre qu'il n'existe aucune forte corrélation entre les variables lorsqu'on se réfère au benchmark de 0,8 (Kennedy, 2003). Par conséquent, il n'existe pas un problème sérieux de multi colinéarité (Kennedy, 2003). Nos variables peuvent donc être mises ensemble dans un modèle régressif.

\subsection{Résultats des régressions Logistique multiples}

Cette partie a pour objectif de vérifier la légitimité des hypothèses de recherche.

Le tableau qui suit présente l'apport de chaque variable indépendante sur la gouvernance au sein des entreprises.

Tableau 5 : synthèse des résultats de régression

Estimation des paramètres des modèles

\begin{tabular}{|c|c|c|c|c|c|c|c|c|c|c|}
\hline & \multirow{3}{*}{$\begin{array}{l}\text { modèle } \\
\text { INST } \\
\text { Wald }\end{array}$} & \multirow{3}{*}{$\begin{array}{l}\text { 2_INV- } \\
\operatorname{Exp}(B)\end{array}$} & \multicolumn{4}{|c|}{ Modèle 1: FREQ CA } & \multicolumn{4}{|c|}{ Modèle 3_EVOL-DUREE AUDIT } \\
\hline & & & \multicolumn{2}{|l|}{ 2fois/an } & \multicolumn{2}{|c|}{ Plus de 2fois/an } & \multicolumn{2}{|c|}{ A la Hausse } & \multicolumn{2}{|c|}{ A la baisse } \\
\hline & & & Wald & $\operatorname{Exp}(\mathrm{B})$ & Wald & $\operatorname{Exp}(B)$ & Wald & $\operatorname{Exp}(B)$ & Wald & $\operatorname{Exp}(B)$ \\
\hline$[\mathrm{ROB}=1]$ & $\begin{array}{l}11,671 * * * \\
(0,001)\end{array}$ & 22,220 & I & I & l & l & $\begin{array}{l}0,02 \\
(0,89)\end{array}$ & 1,103 & $\begin{array}{l}1,557 \\
(0,212)\end{array}$ & 2,859 \\
\hline [CA_SUV=1] & $\begin{array}{l}0,488 \\
(0,485)\end{array}$ & 0,492 & l & l & l & l & $\begin{array}{l}0,07 \\
(0,791\end{array}$ & 0,813 & $\begin{array}{l}0,864 \\
(0,353)\end{array}$ & 2,32 \\
\hline [VIS_CON=1] & $\begin{array}{l}0,979 \\
(0,322)\end{array}$ & 0,329 & $\begin{array}{l}1,345 \\
(0,246)\end{array}$ & 3,035 & $\begin{array}{l}3,519 * \\
(0,061)\end{array}$ & 6,832 & $\begin{array}{l}5,1 * * \\
(0,024)\end{array}$ & 0,675 & $\begin{array}{l}1,835 \\
(0,175)\end{array}$ & 3,987 \\
\hline [ORD_RES=1] & $\begin{array}{l}2,412 \\
(0,12)\end{array}$ & 3,438 & $\begin{array}{l}0,58 \\
(0,446)\end{array}$ & 0,551 & $\begin{array}{l}4,346 * * \\
(0,037)\end{array}$ & 1,184 & $\begin{array}{l}3,17 * \\
(0,075)\end{array}$ & 0,28 & $\begin{array}{l}0,056 \\
(0,812)\end{array}$ & 0,855 \\
\hline [PLA_COM=1] & $\begin{array}{l}0,059 \\
(0,809)\end{array}$ & 0,804 & $\begin{array}{l}1,751 \\
(0,186)\end{array}$ & 3,456 & $\begin{array}{l}\mathbf{3 , 8 3 8} * \\
(\mathbf{0 , 0 5})\end{array}$ & 6,101 & $\begin{array}{l}0,9 \\
(0,344)\end{array}$ & 2,068 & $\begin{array}{l}0,468 \\
(0,494)\end{array}$ & 1,693 \\
\hline [LOG_CON=1] & $\begin{array}{l}13,24 * * * \\
(0,000)\end{array}$ & 2,021 & / & I & l & l & $\begin{array}{l}0,23 \\
(0,632\end{array}$ & 0,637 & $\begin{array}{l}0,228 \\
(0,633)\end{array}$ & 0,643 \\
\hline [SIT_INT=1] & $\begin{array}{l}\mathbf{1 0 , 4 9 * * *} \\
(0,001)\end{array}$ & 1,077 & $\begin{array}{l}0,393 \\
(0,531)\end{array}$ & 1,702 & $\begin{array}{l}0,096 \\
(0,757)\end{array}$ & 0,779 & I & I & I & I \\
\hline [SE_ACT $=1]$ & I & l & $\begin{array}{l}0,195 \\
(0,659)\end{array}$ & 1,783 & $\begin{array}{l}0,358 \\
(0,55)\end{array}$ & 2,174 & l & I & I & I \\
\hline [SE_ACT=2] & I & I & $\begin{array}{l}0,078 \\
(0,78)\end{array}$ & 1,366 & $\begin{array}{l}0,001 \\
(0,971)\end{array}$ & 1,043 & I & I & I & l \\
\hline [SE_ACT=3] & I & I & $\begin{array}{l}1,373 \\
(0,241)\end{array}$ & 0,307 & $\begin{array}{l}0,671 \\
(0,413)\end{array}$ & 0,446 & I & I & l & I \\
\hline EF_PER(1) & $\begin{array}{l}1,763 \\
(0,184)\end{array}$ & 3,069 & I & I & l & l & I & I & I & I \\
\hline EF_PER(2) & $\begin{array}{l}3,498 * \\
(0,061)\end{array}$ & 6,535 & I & I & l & l & l & I & I & I \\
\hline EF_PER(3) & $\begin{array}{l}\mathbf{3 , 1 3 2} * \\
(\mathbf{0 , 0 7 7})\end{array}$ & 0,04 & I & I & l & l & l & I & I & I \\
\hline EF_PER(4) & $\begin{array}{l}0,074 \\
(0,786)\end{array}$ & 0,702 & I & I & I & l & I & I & I & l \\
\hline Constante & 0,018 & 0,703 & 0,319 & & 0,437 & & 0,59 & & 2,034 & \\
\hline
\end{tabular}

Seuil de signification : *** : $\mathrm{p}<1 \% ; * *: \mathrm{p}<5 \% ; *: \mathrm{p}<10 \%$ 
Tableau 6 : estimations spécifiques à chaque modèle

\begin{tabular}{lllll}
\hline Scc ATATISTIQUES & & Modele1 & Modele2 & Modele3 \\
\hline $\mathbf{R}^{2}$ Nagelkerke & & 0,224 & 0,579 & 0,217 \\
& Log-V & 78,607 & & 72,093 \\
Tests des ratios de vraisemblance & Khi2 & 19,364 & 47,25 & 19,133 \\
& p-value & 0,0152 & 0,000 & 0,085 \\
Test Hosmer-Lemeshow & Khi2 & $/$ & 5,142 & $/$ \\
& P-value & $/$ & 0,643 & $/$ \\
\hline \hline
\end{tabular}

Source : Auteur à partir de SPSS version 20

\section{Modèle 1 (Régression sur les fréquences des réunions du conseil d'administration)}

Le modèle estimé permet d'expliquer jusqu'à $22,4 \%$ de la variance de la fréquence des réunions du conseil d'administration par an $\left(R^{2}=0,224\right)$. Vu la p-value du - 2LL (Khi2 =19,364; $\left.p=0,0152\right)$. De ce fait, le modèle est globalement significatif au seuil de $5 \%$.

Les résultats de l'analyse révèlent une influence statistiquement significative des nouvelles technologies sur la fréquence des réunions du conseil d'administration. En effet, les nouvelles technologies augmentent le nombre de séance annuels de conseil d'administration des entreprises. Comparé aux entreprises qui ont tenu un seul conseil entre les membres du conseil d'administration au cours de l'année, on constate qu'aucun indicateur de nouvelle technologie a certes une influence mais non significative sur le nombre de réunions du conseil par an. A contrario, lorsqu'on compare l'entreprise ayant tenu un seul conseil à celle ayant tenu plus de deux conseils au cours de l'année, il se trouve que les indicateurs de la nouvelle technologie contribuent significativement à cette différence de fréquence des réunions du conseil d'administration. En effet, les estimations ont ressortir trois indicateurs de la nouvelle technologie qui différencient significativement l'entreprise ayant tenu un seul entre les membres du conseil d'administration au cours de l'année de celle ayant tenu plus de deux réunions par an. Il s'agit de la Visioconférence, les plateformes collaboratives et la mise en réseau d'ordinateurs. Les statistiques de Wald respectives associé à ces variables $(Z=\mathbf{3 , 5 1 9} ; \mathbf{4 , 3 4 6}$; $\mathbf{3 , 8 3 8}$ ) pour fréquence $=$ plus de deux fois/an sont significatives au seuil de $10 \%$ pour Visioconférence et plateformes collaboratives et $5 \%$ pour ordinateur en réseau $(\mathrm{p}=\mathbf{0 , 0 6 1} ; \mathbf{0 , 0 5 0}$ et $\mathbf{0 , 0 3 7})$. Au regard des rapports de cote, les estimations montrent que l'utilisation de la Visioconférence par l'entreprise, des plateformes collaboratives et la mise en réseau d'ordinateurs augmentent respectivement de $\mathbf{6 , 8 3 2}$ fois, $\mathbf{6 , 1 0 1}$ fois et $\mathbf{1 , 1 8 4}$ fois les possibilités de voir se tenir plus de deux conseils d'Administration par an au sein de l'entreprise.

Au regard de ce qui précède, il est clair que les nouvelle technologies (utilisation des visioconférences, et la mise en réseaux des ordinateurs) ont des effets positifs et statistiquement significative au seuil respective de $\mathbf{1 0 \%}$ et $\mathbf{5 \%}$ sur la fréquence du conseil d'administration. Dès lors hypothèse (H1) est 
validée. Ce qui corrobore travaux de Valerie, (2018), qui conclut que les nouvelles technologies ont un impact significatif sur la fréquence des réunions du conseil d'administration. De plus, ce résultat rejoint les travaux de Kauffman et Riggins (2012) qui ont trouvé que dans un environnement concurrentiel, les technologies numériques permettent aux IMF de garantir leur performance tout en augmentant la fréquence des réunions du conseil d'administration de veiller à la mauvaise gouvernance et surtout leur survie.

\section{Modèle 2 (Régression sur l'investisseur institutionnel)}

Au regard de la $\mathrm{p}$-value $(\mathrm{p}=0.000)$, de la différence entre les ratios de vraisemblance de l'étape 0 et l'étape final des estimations $(-2 \mathrm{LL}=\mathrm{Khi} 2=47,25)$, il apparait que ce modèle est globalement significatif, c'est à dire qu'il existe au moins une variable indépendante qui exerce une influence significative sur la variable dépendante. Par ailleurs, les termes de l'équation logistique finale prédisent mieux la probabilité d'attirer les investisseurs institutionnels que ne le fait la probabilité initiale observée. En effet, le test de Hosmer-Lemes-how nous indique que les valeurs prédites et observées sont cohérentes $(\mathrm{p}=0,643>5 \%)$; donc qu'il n'existe pas une différence significative entre les valeurs prédites et observées dans notre modèle final. Le modèle estimé est d'une robuste acceptable, il permet d'expliquer jusqu'à $57,9 \%$ de la variance de la présence d'investisseurs institutionnels au sein des entreprises $\left(R^{2}\right.$ de Nagelkerke $\left.=0,579\right)$.

Les tests effectués révèlent l'existence d'un lien significatif fort et positif entre l'intelligence artificielle et la présence des investisseurs au sein de l'entreprise. En effet, nous pouvons constater en ce qui concerne la variable Robotique que les estimations renvoient un coefficient positif $(\mathbf{3 , 1 0 1})$ et la statistique de Wald $(\mathrm{Z}=\mathbf{1 1 , 6 7 1})$ est significatif au seuil de $1 \%$ ( $\mathrm{p}$-value=0,001). Le rapport de cote associé est de 22,22. Ce résultat signifie que la chance de trouver une entreprise où les investisseurs institutionnels sont présents augmente de 22,22 fois lorsque cette entreprise a un système en Robotique.

Cependant, les estimations révèlent une relation non significative entre le Blockchain et l'entrée des investisseurs institutionnels au sein de l'entreprise.

Lorsqu'un observe les variables LOG_CON(1) et SIT_INT(1), on constate que leur statistique de Wald respective $(Z=\mathbf{1 3 , 2 4 0}$ et 10,492) sont toutes significative au seuil de $1 \%$ (p value $=0,000$ et 0,001 ). Les rapports de cote respectifs sont de $\mathbf{2 , 0 2 1}$ et $\mathbf{1 , 0 7 7}$. Des lors, on peut conclure que comparé à une entreprise qui ne possède pas de logiciel de contrôle de gestion et un site internet, les chances d'attirer les investisseurs institutionnels augmente de respectivement de $\mathbf{2 , 0 2 1}$ fois et de $\mathbf{1 , 0 7 7}$ fois lorsque l'entreprise à des logiciels de contrôle de gestion et un site internet. 
Les développements ci-dessus, montrent de façon globale que l'intelligence artificielle (robotique) et le Big Data (site internet et logiciel de contrôle de gestion) favorise la présence des investisseurs institutionnels au sein de l'entreprise. De ces résultats nous pouvons dire que l'hypothèse 2 est validée. Ce résultat rejoint les travaux de (Mathur et Ambani, 2013), qu'ils conclurent que l'utilisation des technologies numériques permet de réduire les risques et d'améliorer la transparence dans la gestion des entreprises afin d'attirer l'attention des investisseurs institutionnels. Ces derniers estiment que l'adoption des technologies numériques par les IMF en plus de contribuer à la réduction de leur fracture numérique, leur permet d'exercer avec plus d'efficience et d'efficacité. Selon Kaal (2017), la technologie blockchain pourrait conduire à un modèle de gouvernance décentralisée dans lequel les mécanismes classiques de contrôle interne et externe nécessaires pour résoudre les problèmes des agences ne seraient plus nécessaires. La technologie blockchain pourrait offrir une solution au problème des agences et à ses coûts connexes (Lafarre et Van der Elst, 2018).

\section{$>$ Modèle 3 (Régression sur l'auditeur externe)}

Le modèle estimé est d'une robuste non négligeable, il permet d'expliquer jusqu'à 21,7\% de la variance de l'évolution de la durée des missions d'audit $\left(\mathrm{R}^{2}=0,217\right)$. Vu la p-value de la différence du ratio de vraisemblance des estimations de l'étape 0 et l'étape finale (Khi2 =19,133; p=0,085), le modèle est globalement significatif au seuil de $5 \%$.

Les résultats de l'analyse révèlent une influence statistiquement significative des nouvelles technologies sur la durée de mission de l'auditeur externe. On constate que grâce à les nouvelles technologies la durée de mission de l'audit externe augment. En effet, comparé aux entreprises qui ont une durée de mission d'audit externe en hausse on constate que les indicateurs des nouvelles technologies ont une influence négative et statistiquement significative sur la durée de la mission de l'audit externe. A contrario, lorsqu'on compare l'entreprise qui confirme que la durée de mission de l'audit est en baisse, il se trouve que les indicateurs des nouvelles technologies présente une relation mitigée (une influence positive et négative) et n'est pas significative à cette durée de mission de l'audit externe. En effet, les estimations ont ressortir deux indicateurs des nouvelles technologies qui différencient significativement des entreprises qui confirment que la durée de mission de l'audit est à la hausse et en baisse. Il s'agit de la Visioconférence, et la mise en réseau des ordinateurs. Les statistiques de Wald respectives associé à ces variables $(Z=\mathbf{5 , 1}$; et $\mathbf{3 , 1 7})$ pour durer de mission d'audit = à la hausse sont significatives au seuil de $\mathbf{5 \%}$ pour Visioconférence et $\mathbf{1 0 \%}$ pour ordinateur en réseau $(p=\mathbf{0 , 0 2 4}$ et $\mathbf{0 , 0 7 5}$ ). Au regard des rapports de cote, les estimations montrent que l'utilisation par l'entreprise de la Visioconférence et la mise en réseau d'ordinateurs augmente respectivement de $\mathbf{0 , 6 7 5}$ et $\mathbf{0 , 2 8}$ la dure de mission de l'audit. On peut conclut qu'une entreprise qui instaure l'utilisation de la visioconférence pour tenir des réunions contrairement à celle qui ne dispose pas la durée de mission augment. 
Contrairement à nos anticipations on constate que les nouvelles technologies (utilisation des visioconférences et la mise en réseaux des ordinateurs) ont une influence négativement et statistiquement significative au seuil respective de $\mathbf{5 \%}$ et $\mathbf{1 0 \%}$ sur la durée de mission de l'audit externe. Ce qui permet de rejeter l'hypothèse $(\mathrm{H} 3)$ formuler au départ de la manière suivante « Les nouvelles technologies réduisent la durée de mission de l'audit externe.» ce qui est contraire aux travaux de Wallace, (2004) ; Andon et al. (2014); Jeacle, (2014, 2017 ),_soutiennent que l'utilisation des nouvelles technologies facilite la vérification des comptes de l'entreprise et réduit le temps de vérification. Najoua et Baudier(2017) affirment que l'utilisation des technologies numériques au sein de l'entreprise influence positivement sur la durée de mission de l'auditeur externe.

\section{Conclusions}

Une panoplie de solutions technologiques a été expérimentée en entreprise dans l'optique d'améliorer sa gouvernance, allant des plus élémentaires aux plus complexes (Ivatory, 2006). Rappelons que l'objectif de cet article était de mesurer l'effet des nouvelles technologies sur le conseil d'administration, la structure de propriété et l'auditeur externe au Cameroun. Pour y parvenir, plusieurs instruments méthodologiques ont été mobilisés : le tri à plat a permis d'expliquer la répartition statistique des composantes de l'échantillon à travers les différentes variables étudiées. Le test de Khi-2 a facilité la compréhension de la dépendance entre les caractéristiques des nouvelles technologies et gouvernance d'entreprise. Enfin, la régression logistique multinomiale a été utilisée pour mesurer l'influence réelle de l'utilisation des nouvelles technologies sur la gouvernance d'entreprise. Cette technique relève de façon globale un impact positif et significatif au seuil respectif de $10 \%$ et $5 \%$ des nouvelles technologies sur la fréquence des réunions du conseil d'administration. Par ailleurs, lorsque les nouvelles technologies sont appréhendés par l'utilisation des logiciels de contrôles et la présence un site internet, on observe des effets positive et significative au seuil de $1 \%$ sur la présence des investisseurs institutionnels. Par contre, lorsqu'elles sont appréhendées par l'utilisation des visioconférences et la mise en réseaux des ordinateurs l'influence est plutôt négative et significative aux seuils respectifs de $5 \%$ et $10 \%$ sur la durée de mission de l'audit externe.

\section{BIBLIOGRAPHIE}

[1] Akter, S., \& Wamba, F., (2016). « Big data analytics in e-commerce »: asystématic Review and agenda for future Research Electronic Markets, 26 (2), 173-194.

[2] Anderson, C. (2014). « Makers : The New Industrial Revolution », Crown Business.

[3] Ashta, A., \& Assadi, D., (2008), « Do Social Cause and Social Technology Meet? Impact of Web 2.0 Technologies on Peer-to-peer Lending Transactions », Burgundy School of Business, CEREN

[4] Bebchuk, A., Cohen, A., \& Hirst, S., (2017). « Les problèmes d'agence des investisseurs institutionnels ». Journal of Economic Perspectives, 31(3), 89- 102. https://doi.org/10.1257/jep.31.3.89

[5] Beckhard R., \& Dyer W., (1983), « Managing Change in the Family Firm - Issues and Stratégies », Sloan Management Review, 24(3), 59-65.

[6] Botsman, R., (2017). «À qui pouvez-vous faire confiance ? Comment la technologie nous a rapprochés et pourquoi elle pourrait nous séparer. Londres, Royaume-Uni : Portfolio Penguin ». 
[7] Braune, E., Lantz JS., Sahut, JM. \&Teulon, F. (2019). « Le capital-risque d'entreprise dans le secteur des TI et les relations dans les réseaux de syndication du capital-risque ». Small Business Economics, Première publication : 29 août 2019, https://doi.org/10.1007/s11187-019-00264-4

[8] Buterin, V., (2014). DAO, DAC, DA et autres : «Un guide terminologique incomplet». Consulté sur https://blog.ethereum.org/2014/05/06/daos-dacs-das- and-more-an-incomplete-terminology-guide/

[9] Caby, J., \& Hirigoyen, G., (2002), « La gestion des entreprises », Paris, Economica.

[10] Caron. F., (1997), « Les deux révolutions industrielles du XX siècle », Albin Michel, Paris, 12-23.

[11] Cassiman, B., \& Veugelers, R., (2002), «Complementary in the innovation strategy: Internal R et D, external technology acquisition and coopération in $\mathrm{R}$ et $\mathrm{D} »$. Research paper 457, 2002, Barcelona, Spain : University of Naavarre, IESE.

[12] Charreaux, G., (2002), « Variation sur le thème: à la recherche de nouvelle fondation pour la finance d'entreprise ». Finance contrôle et stratégies, 92(3), 5-68.

[13] De Massis, A., Frittini F., \&Lichtenthaler, U., (2013), «Reseach on technological innovation in family films: present Debates and Futur Directions ». Business Review, 26 (1), 10-31.

[14] El Bouame, F. \& Lempqeddem H., (2021), «Impact de l'innovation sur la croissance économique : réflexion théorique du Nexis entre les deux concepts », vol 2, $\mathrm{n}^{\circ} 4$.

[15] Enriques, L., \& Zetzsche, D., (2019). « Corporate technologies and the tech nirvana fallacy (European Corporate Governance Institute (ECGI) ». Law Working Paper 457/2019). Consulté sur https://ssrn.com/abstract=3392321.

[16] Erel, I., Stern, H., Tan, C., \& Weisbach, S., (2018). « Selecting directors using machine learning (NBER Working Paper No.24435) ». Consulté sur https://www.nber.org/papers/w24435.

[17] Erickson Gary M., \& Jacobson Robert, (1992), « Gaining competitive advantage through discretionary expenditure: returns to R and D and advertising ». Management science, 38, 1264-1279.

[18] Esposito De Falco, S., Cucari, N., Canuti, E., \& Modena, S., (2019). « Gouvernement d'entreprise et Blockchain : Quelques résultats préliminaires d'une enquête ». Gouvernance d'entreprise : Search for the Advanced Practices, 102-115. https://doi.org/10.224 95/cpr19p3.

[19] Fambeu, H., (2017), «L'adoption des TIC dans un pays en développement », Revue d'économie industrielle, 1, 61101.

[20] Fichman, G., Dos Santos, L., \& Zheng, Z., (2014). «Innovation numérique en tant que concept fondamental et puissant dans le programme d'étude des systèmes information ». MIS Quarterly 38 (2), $329-343$.

[21] Granlund \& Malmi T., (2002) «moderate impact of ERPS on management accouting »A lag or permanent outcome? Management Accounting Research, 13, 185 -211. Management Accounting Research 31, 118-122.

[22] Hamdani, A., Hashai, N., Kandel, E. \& Yafeh, Y., (2019). « Processus technologique et avenir de l'entreprise ». Journal of the British Academy, 6(1), 215-245. https://doi.org/10.5871/jba/006s1.215.

[23] Hartarska, V., \& Nadolnyak, D., (2008), « Does Rating help Microfinance Institutions Raise Funds? », International Review of Economics and Finance, 17, 558-571.

[24] Hirigoyen G., (2000), « le Gouvernement des entreprises familiales », article publié dans le rapport moral sur l'argent dans le monde, Montchrestien, 287-294.

[25] Hirigoyen G., (2002), « Le gouvernement des entreprises, in La gestion des entreprises », sous la direction de Caby J. et Hirigoyen G. Economica.

[26] Iansiti, M., \& Lakhani, R., (2017). «La vérité sur Blockchain ». Consulté sur https://hbr.org/2017/01/the-truthabout-blockchain.

[27] Imai, K., Arun, T., \& Annim, S., (2010), «Microfinance and Household Poverty Reduction: New Evidence from India », World Development, 38(12), 1760-1774.

[28] Ivatory, G., (2006), « Using Technology to Build Inclusive Financial Systems », CGAP Focus Note 32, January, Washington DC.

[29] Jensen, C., \& Meckling, H., (1976). «Théorie de l'entreprise : Comportement des dirigeants, coûts des agences et structure de propriété ». Journal of Financial Economics, 3(4), 305-360. https://doi.org/10.1016/0304- 405X (76)90026-X.

[30] Joseph Schumpeter, (1934), « theory of economic development Harvard University press », Cambridge, 148-156.

[31] Kaal, A., (2017). «Blockchain solutions for agency problems in corporate governance». Consulté sur https://medium.com/@wulfkaal/blockchain-_ solutions-for-agency-problems-in-corporate-governancea83aae03b846.

[32] Kaal, A., Dell'Erba, \& M., (2017). «Blockchain innovation in private investment funds» - A comparative analysis of the United States and Europe (U of St. Thomas Legal Studies Research Paper, 17-20). Consulté sur https://ssrn.com/abstract=3002908. 
[33] Kelly, K. (2016). «L'inévitable : Comprendre les 12 forces technologiques qui façonneront notre avenir, Viking ».

[34] Kohli, R., \& Melville, P., (2018) « innovation numérique : examen et synthèse ». Information sytems journal 29 (1), $200-223$.

[35] Lajmi A., (2005), «TIC et nouveau rôle du contrôle de gestion » le contexte des entreprise tunisiennes, cahier électronique, institut supérieur de gestion de Tunis.

[36] Lichtenthaler \& Muethel M., (2012). "The impact of family involvement on dynamic innovation capability » evidence from German manufacturing firms. Entrepreneurship theory and practice, 36 (6), 1235-1253.

[37] Lyytinen, Yoo, Y., \& Boland J., (2016). «Innovation des produits numériques au sein de quatre classes de réseau innovation ». Information systems journal, 26(1) 47-75.

[38] Nambisan, S., Lyytinen, K. Majchrzak, A. \& Song, M., (2017). « Recherché dans un monde numérique ». MIS \& Quarterly 41 (1), 23-28.ù*ù*, «an evolution theory of economic change Cambridge M.A». Harvard University press.

[39] Nikishova, M., (2017) «Perspectives d'utilisation de l'intelligence artificielle dans la gouvernance d'entreprise dans le contexte de la transition vers une économie numérique ». V Conférence internationale sciences de gestion dans le monde moderne // Amélioration du système de gouvernance d'entreprise : Nouvelles approches, technologies et outils. 2017. URL : http://www.management- science.ru/works-digest/.

[40] OCDE, (1997), «La mesure des activités scientifique et technologique » : principes directeurs proposes pour les recueils des donnes sur l'innovation technologique manuel d'Oslo, Ed. De L'OCDE et Eurostat.

[41] OCDE, (2004), « Principes de gouvernement d'entreprise ».

[42] Porter miachal, (1986), «L'avantage concurentiel », Dunod, paris.

[43] Quattrone, P., (2016), «Management Accounting goes digital»: Will the move make it wiser?

[44] Rongé Y., (2006) «l'impact des ERP sue le contrôle de gestion une première évaluation », fineco 10, 45 -65. http://wwwfsa.Ca/ fineco / Yves - de - Ronge. PDF.

[45] Sahut, JM, Dana, LP \& Laroche, M. (2019). «Innovations numériques, impacts sur le marketing, la chaîne de valeur et les modèles commerciaux : Une introduction ». Revue canadienne des sciences administratives ; Première publication : 17 novembre 2019, https://doi.org/10.1002/cjas.1558

[46] Sahut, JM., Iandoli, L. \& Teulon, F. (2019). L'ère de l'entreprenariat numérique. Small Business Economics, Première publication : 9 septembre 2019, https://doi.org/10.1007/s11187-019-00260.

[47] Solow, R.M. (1987). « Il faut faire attention ». New York Times Book Review, 36.

[48] Tsafack R., (2002), « NTIC et développement Africain » in Revue Africaine des Sciences Économiques et Gestion, 6(1), 3-25.

[49] Van de Ven A., \& Poole M., (1995), « explaining development and change in organization », the academy of management Review, 20(3), 510-539.

[50] Varis M. \& Littunen. H., (2010), « Types of innovation, sources of information and performance in entrepreneurial SMEs ». European Journal of Innovation Management, 13(2), 128-154.

[51] Vasarhelyi, A., Kogan, A., \&Tuttle, M., (2015). «Big Data in Accounting»: An Overview.

[52] Wernerfelt B., (1984), «A Ressource-based View of The Firm», Strategic Management Journal, 180 pages.

[53] Wiesbock, F., (2019), «Innover dans un monde numériques, le rôle des capacités innovations des produits numériques ». Document présenté lors de la $27 \mathrm{eme}$ conférence europeenne sur le systeme information, StockholmUppsala, Sued.

[54] www.wikipidia.org.la financepourtous.com 